\title{
Implant supported fixed partial dentures survival rate high, but biological and technical complications common
}

\author{
How long do implant supported fixed partial dentures (FPDs) survive and how \\ frequently do complications occur?
}

\begin{abstract}
Pjetursson BE, Tan K, Lang NP, Bragger U, Egger M, Zwahlen M. A systematic review of the survival and complication rates of fixed partial dentures (FPDs) after an observation period of at least 5 years - I. Implant-supported FPDs. Clin Oral Implants Res 2004 Dec; 15:667-676.
\end{abstract}

Data sources PubMed (1966-April 2004) provided the primary data source along with the bibliographies from identified articles and reviews. Manual search of eight relevant journals (published 20012003) provided a further source of data.

Study selection As there were no randomised controlled trials comparing implant therapy with conventional reconstructive dentistry, English language prospective and retrospective cohort studies were selected if; they had a mean follow-up of $\geq 5$ years; included patients who were clinically examined at follow-up; reported details on suprastructures and described at least one-third of reconstructions as fixed partial dentures (FPDs).

Data extraction and synthesis Two independent reviewers screened articles for inclusion. Disagreements were resolved by discussion and agreement determined by kappa. Data were extracted on the survival proportions of the reconstructions and on biological and technical complications. Biological complications included disturbances in the function of the implant characterized by a biological process affecting the supporting tissues. Technical complications denoted mechanical damage of implants, implant components, or the suprastructures. The number of events for all categories was identified and the corresponding total exposure time of the reconstruction calculated. Failure and complication rates were calculated by dividing the number of events by the total exposure time.

Results Twenty-one studies of implant supported FPDs, reporting on 16 patient cohorts were included. Of the studies, 16 were prospective and five retrospective. A total of 1336 FPDs supported by 3578 oral implants were involved. Meta-analyses gave an estimated implant survival of $95.4 \%$ (95\% Cl 93.9-96.5) after 5 years and $92.8 \%$ (95\% Cl 90-94.8) after 10 years. Implant failure rate was $48 \%$ lower $(P=0.006)$ in the more recent studies. The estimated survival for the FPDs was 95\% (95\% Cl 92.2-96.8) after 5 years and $86.7 \%(95 \% \mathrm{Cl}$ 82.8-89.8) after 10 years. Four studies provided information on the number of patients free from complications - after 5 years $61.3 \%$ (95\% Cl 55.3-66.8) had suffered no minor or major complications. A random effects Poisson model revealed the rate of biological complications for patients treated with implant supported FPDs to be $8.6 \%(95 \%$ Cl 5.1-14.1) after 5 years. For technical complications the cumulative 5 year complication rate varied from $0.4 \%$ for implant fracture to $13.2 \%$ for veneer fracture.

Conclusions Although survival rate for implant supported FPDs is high, biological and technical complications are relatively common.

Address for correspondence: Bjarni E Pjetursson, Department of Periodontology and Fixed Prosthodontics, University of Berne Freiburgstrasse 7, CH 3010 Berne, Switzerland. E-mail: bjarni.pjetursson@zmk.unibe.ch

\section{Commentary}

This systematic review is part of a series of systematic reviews, by the same group, ${ }^{1-4}$ addressing the survival and complication rates of fixed partial dentures of different designs.

This review had a clear objective; to assess the 5- and 10-year survival of implant supported fixed partial dentures and to describe the incidence of biological and technical complications. Sufficient evidence exists to support the view that implants provide a predictable and long-term mode of therapy for edentulous patients. However data on fixed partial dentures is still relatively sparse and short-term. More such data are required to assist clinicians and patients alike in decision making, thus the paper discussed here is of value.

The authors conducted a thorough search of the literature; however, this was limited to papers published in the English language only. The authors make the point that a few studies in other languages were identified. It is impossible to judge whether or not the patient cohorts excluded from the present review might have differed in terms of survival and complication rates.

Of the 21 that met the clearly defined inclusion criteria there were no randomised controlled clinical trials available so the data presented are from prospective and retrospective cohort studies, all published within the last 10 years. Data from a total of 3578 dental implants and 1336 FPDs were analysed, of which 299 FPDs were analysed both after a follow-up time of 5 and 10 years.

The survival rate of FPDs supported by implants was 95\% after 5 years and $86.7 \%$ after 10 years. With implant survival rates of $95.4 \%$ at 5 years and $92.8 \%$ at 10 years similar to the results from a previous systematic review ${ }^{5}$ and other traditional reviews. ${ }^{6}$ Periimplantitis and soft tissue complications occurred in $8.6 \%$ of FPD's after 5 years. After 5 years, the cumulative incidence of connectionrelated complications (screw-loosening or fracture) was $7.3 \%$, and $14 \%$ for suprastructure-related complications (veneer and framework fracture).

The authors draw the conclusion that despite the high survival of FPDs, biological and technical complications are frequent. This has implications, in that a substantial amount of chair-time has to be accepted by the patient and provided for by the dental service provider, following the incorporation of implants supporting FPDs.

The studies within this review were mainly conducted in institutional environments and the long-term outcomes observed may not be generalisable to dental service provision in a practice setting. Even with follow-up periods of at least 5 years, some may argue that this is too short a time period, to obtain reliable information on survival and complication rates. As the authors point out, clearly there is a need for more longterm studies with well-defined criteria for the assessment of complications. Furthermore more information is required on how these restorations perform in all types of clinical environment settings. 


\section{Practice point}

- While implant supported FPDs show good survival frequent biological and technical complications have implications for patients and providers.

\section{Pranay Sharma}

Eastman Dental Institute, University College London, 256 Gray's Inn Road, London, UK

1. Pjetursson BE, Tan K, Lang NP, Bragger U, Egger M, Zwahlen M. A systematic review of the survival and complication rates of fixed partial dentures (FPDs) after an observation period of at least 5 years -IV. Cantilever or extension FPDs. Clin Oral Implants Res 2004; 15:625-642.

2. Lang NP, Pjetursson BE, Tan K, Bragger U, Egger M, Zwahlen M. A systematic review of the survival and complication rates of fixed partial dentures (FPDs) after an observation period of at least 5 years. II. Combined tooth-implant-supported FPDs. Clin Oral Implants Res 2004; 15:643-653.
3. Tan K, Pjetursson BE, Lang NP, Chan ES. A systematic review of the survival and complication rates of fixed partial dentures (FPDs) after an observation period of at least 5 years- III. Conventional FPDs. Clin Oral Implants Res 2004; 15:654-666.

4. Pjetursson BE, Tan K, Lang NP, Bragger U, Egger M, Zwahlen M. A systematic review of the survival and complication rates of fixed partial dentures (FPDs) after an observation period of at least 5 years- I. Implant-supported FPDs. Clin Oral Implants Res 2004; 15:667-676.

5. Berglundh T, Person L, Klinge B. A systematic review of the incidence of biological and technical complications in implant dentistry reported in prospective longitudinal studies of at least 5 years. J Clin Periodontol 2002; 29(Supp. 3): 197-212.

6. Esposito M, Hirsch J, Lekholm U, Thomsen P. Biological factors contributing to failures of osseointegrated implants I. Success criteria and epidemiology. Eur J Oral Sci 1998; 106:527-551.

Evidence-Based Dentistry (2005) 6, 72-73.

doi:10.1038/sj.ebd.6400349 